\title{
Isolierung eines neuen Insektenhormons aus Seidenspinnerpuppen
}

\author{
Hans Hoffmeister \\ Physiologisch-Chemisches Institut der Universität Marburg/L., und \\ I. Medizinische Universitätsklinik, Hamburg \\ (Z. Naturforschg. 21 b, 335-336 [1966] ; eingegangen am 14. Dezember 1965)
}

\begin{abstract}
Die Gewinnung eines weiteren Insektenhäutungshormons aus Extrakten von Seidenspinnerpuppen wird beschrieben. Das Hormon - Ecdysteron - verhält sich chromatographisch polarer als das schon bekannte Ecdyson. Ecdysteron besitzt im Calliphoratest eine stärkere Wirksamkeit als Ecdyson. Aus 2 to Seidenspinnerpuppen wurden $9 \mathrm{mg}$ Hormon erhalten.
\end{abstract}

1954 isolierten Butenandt und $\mathrm{KarLson}^{1}$ als erstes kristallisiertes Insektenhormon das Ecdyson, ein Häutungs- und Metamorphosehormon. Seine Strukturaufklärung ergab, daß es sich um ein Steroidhormon handelt ${ }^{2,3}$. Neben dem Ecdyson vom Schmp. $239^{\circ}-242^{\circ}$ wurde später noch ein ähnliches Kristallisat isoliert, das allerdings bisher nur durch den Schmelzpunkt $\left(177^{\circ}\right)$, das UV- und IRSpektrum und die biologische Aktivität charakterisiert wurde ${ }^{4}$. Burdette und Bullock ${ }^{5}$ beschrieben eine Auftrennung eines Rohextraktes in fünf aktive Fraktionen, ohne allerdings reine Substanzen zu isolieren. Über ein weiteres kristallisiertes Häutungshormon, das Ecdysteron, wurde kürzlich in einer vorläufigen Mitteilung berichtet ${ }^{6}$. Ecdysteron konnte aus Extrakten von Bombyx-mori-Puppen gewonnen werden. Das Hormon ist im Calliphoratest etwa 2 -3-mal so aktiv wie Ecdyson. 0,003-0,005 $\mu \mathrm{g}$ Ecdysteron entsprechen einer Calliphora-Einheit.

Als Ausgangsmaterial für die Isolierung dienten die Extrakte von Seidenspinnerpuppen, aus denen vorher das Ecdyson isoliert worden war. Die Aufarbeitung auf Ecdysteron geschah in der nachfolgend beschriebenen Weise:

2 to Seidenspinner-Trockenpuppen, wie sie nach Abspulen der Seidenkokons anfallen, wurden gemahlen und mit Methanol extrahiert. Der Methanolrückstand wurde mit Butanol extrahiert; nach Verdampfen des Butanols erhält man einen Sirup, der sich beim Verteilen zwischen Petroläther und Wasser teilweise in Wasser löst. Die Wasserphase enthält die Hauptmenge der vorhandenen Häutungs-

1 A. Butenandt u. P. Karlson, Z. Naturforschg. 9 b, 389 [1954].

2 P. Karlson, H. Hoffmeister, H. Hummel, P. Hocks u. G. Spiteller, Chem. Ber. 98, 2394 [1965].

3 R. Huber u. W. Hoppe, Chem. Ber. 98, 2403 [1965].

4 P. Karlson, Ann. Sci. natur., Zool. Biol. animale 18, 125 [1956].
hormon-Aktivität, wie die Auswertung der Extrakte im Calliphora-Test ${ }^{7}$ ergab. Die in Wasser gelöste Substanz enthielt $50 \mathrm{CE} / \mathrm{mg}$. Durch Chromatographie an $\mathrm{Al}_{2} \mathrm{O}_{3}$ und Gegenstromverteilung wurde aus diesem Extrakt Ecdyson gewonnen ${ }^{8}$. Alle Fraktionen, die nach den einzelnen Schritten der Ecdysonaufarbeitung übrig blieben, wurden vereinigt. Die verbliebene Aktivität des Extraktes im Calliphoratest betrug $6 \mathrm{CE} / \mathrm{mg}$. Das entspricht $12 \%$ der vor Entfernung von Ecdyson vorhandenen Häutungshormon-Aktivität.

Die Isolierung des Ecdysterons wurde nach dem folgenden Schema vorgenommen: Der zähe Sirup wurde mit Essigester extrahiert. Die Essigesterauszüge enthielten die Hauptmenge der Häutungshormon-Aktivität. Der Essigesterrückstand wurde über eine $\mathrm{Al}_{2} \mathrm{O}_{3}$-Säule mit einem Lösungsmittelgradienten aus Methanol und Benzol aufgetrennt (Abb.1).

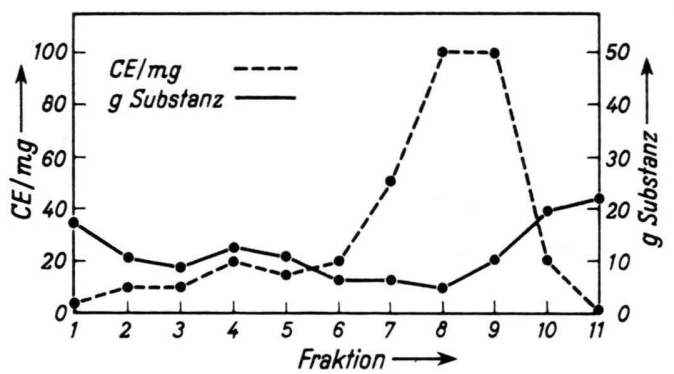

Abb. 1. Säulenchromatographie von $150 \mathrm{~g}$ Bombyx-Extrakt an $3 \mathrm{~kg} \mathrm{Al}_{2} \mathrm{O}_{3}$, Woelm, Aktivitätsstufe III. Frakt. 1: $6 l$ Benzol; Frakt. 2: $6 l$ Benzol 9/Methanol 1; Frakt. 3: $6 l$ Benzol 8/Methanol 2 usw. bis Frakt. 11: $21 l$ Methanol.

5 W. J. Burdette u. M. W. Bullock, Science [New York] 140, 1311 [1963].

6 H. Hoffmeister, Angew. Chem. 78, 269 [1966].

7 P. Karson, Vitamins and Hormones 14, 227 [1956].

8 P. Karlson, H. Hoffmeister, W. Hoppe u. R. Huber, Liebigs Ann. Chem. 662, 1 [1963]. 
Ein Anteil jeder Fraktion wurde auf 1,5 mm dicken Kieselgelschichten chromatographiert. In den verwendeten Lösungsmittelsystemen wanderten unpolare Steroide, wie Cholesterin oder Pflanzensterine, an der Front. Die einzelnen Fraktionen der Säulenchromatographie wurden auf ihren Gehalt an polaren Steroiden untersucht. Sehr viele Steroide lassen sich mit Vanillin/Schwefelsäure anfärben. Diese Reaktion wurde benutzt, um Steroidbanden auf den einzelnen Kieselgelschichten nachzuweisen.

Die Fraktionen 7, 8 und 9 der Säulenchromatographie zeigten im Schichtchromatogramm an Kieselgel eine schwach türkis gefärbte Bande um den $R_{f^{-}}$ Wert 0,1, System $\mathrm{CH}_{2} \mathrm{Cl}_{2}$ 80/Äthanol 25/Aceton 20. Die gesamte Substanzmenge der drei Fraktionen wurde dann auf Kieselgelschichten getrennt. Die im UV-Licht erkennbaren Banden wurden extrahiert und im Calliphoratest der Häutungshormon-Gehalt festgestellt. Die Banden zwischen den $R_{f}$-Werten 0,05 und 0,3 zeigten Hormonaktivität. Aus dieser Zone konnten $260 \mathrm{mg}$ Substanz gewonnen werden.

Die weitere Anreicherung der aktiven Substanz gelang durch eine erneute Chromatographie an Kieselgelschichten. Im System Isoamylalkohol 75 /Äthanol 5 wurde nach doppelter Entwicklung der Platten eine scharfe Zone erhalten, die im UV-Licht absorbierte. Die Bande färbt sich mit Vanillin/Schwefelsäure rosa an, die Farbe geht beim Erwärmen in Türkis über. Aus dem hellbraunen Rohextrakt ließen sich durch Umkristallisieren aus Wasser und Essigester/Methanol $9 \mathrm{mg}$ farblose Kristalle erhalten; Schmp. $226^{\circ}$. Die Substanz absorbiert im $\mathrm{UV}, \lambda_{\max }=240 \mathrm{~m} \mu, \varepsilon=13600$. Im IR-Spektrum liegt die Carbonylbande bei $1645 \mathrm{~cm}^{-1}$ und die dazu konjugierte Doppelbindungsbande bei $1612 \mathrm{~cm}^{-1}$ (Aufnahme in $\mathrm{KBr}$ ). Die Kristalle lösen sich leichter in Wasser als Ecdyson. Sie haben im CalliphoraTest eine Aktivität von $1 \mathrm{CE} / 0,003-0,005 \mu \mathrm{g}$ (Ecdyson: $1 \mathrm{CE} / 0,01 \mu \mathrm{g})$.

\section{Experimenteller Teil}

$410 \mathrm{~g}$ wäßriges Konzentrat nach der Abtrennung von Ecdyson (s. allgem. Teil) wurden im Wasser- bad erwärmt, mit $2 l$ Essigester überschichtet und unter Rückfluß 12 Stdn. gerührt. Die Essigesterschicht wurde abgegossen. Zweimaliges Wiederholen der Ausrührung erbrachte insgesamt $150 \mathrm{~g}$ durch Essigester extrahierbares Material. Der zähe, dunkelbraune Sirup wurde an $3 \mathrm{~kg} \mathrm{Al} \mathrm{O}_{3}$, Woelm, Aktivitätsstufe 3 , chromatographiert. Elution mit einem Lösungsmittelgradienten aus Benzol und Methanol ergab die in Abb. 1 aufgeführten Fraktionen. Die Fraktionen 7, 8 und 9 wurden vereinigt und auf 30 Schichtchromatographie-Platten $20 \cdot 20 \mathrm{~cm}$ mit 1,5 mm dicken Kieselgelschichten aufgetrennt. Kieselgel HF, Merck, Laufmittel 1 = Methylenchlorid 80/Äthanol 25/Aceton 20. Im UV-Licht waren etwa 10 verschieden starke Banden auf den Platten zu erkennen. Der Bereich von $R_{f}$-Wert $0,05-0,3$ wurde 2 Tage lang im Soxhlet-Apparat mit Methanol extrahiert. Nach Eindampfen der Methanolauszüge bis auf $100 \mathrm{ml}$ ließ sich ein großer Teil des gelösten Kieselgels durch 2-stdg. Zentrifugieren bei $15000 \times g$ entfernen. Der Methanolüberstand enthielt 1,8 g Rückstand, der erneut an Kieselgel im Laufmittel 1 chromatographiert wurde. Eine im UV-Licht schwach erkennbare Bande um $R_{f}$-Wert 0,1 färbte sich beim Besprühen mit einer Lösung aus 5 Tln. konzentrierter Schwefelsäure und 1 Tl. 25-proz. alkoholischer Vanillin-Lösung in der Kälte rosa an; beim Erwärmen auf $110^{\circ}$ ändert sich die Farbe in Türkis.

Die Zone wurde wie oben beschrieben behandelt und ergab $420 \mathrm{mg}$ braunen, zähen Rückstand. In einer weiteren Schichtchromatographie an Kieselgel konnte die mit Vanillin/Schwefelsäure anfärbbare Substanz weiter angereichert werden: Laufmittel 2 =iso-Amylalkohol 75/Äthanol 5. $R_{f}$-Wert nach zweifacher Entwicklung 0,6. Mit Methanol ließen sich $56 \mathrm{mg}$ hellgelbes Öl extrahieren. Nach längerem Stehen kristallisierten daraus große, farblose Prismen. Durch Umkristallisieren aus wenig Wasser und anschließend aus Essigester/Methanol ließen sich $9 \mathrm{mg}$ des reinen Hormons gewinnen.

Herrn Prof. Dr. P. Karlson danke ich für die Förderung dieser Arbeit sowie für die Durchsicht des Manuskripts, der Deut s chen Fors chung s e mein$\mathrm{s} \mathrm{ch}$ a f $\mathrm{t}$ für finanzielle Unterstützung. 